Running Head: Mother-child relationship positivity and negativity interact

(C) 2017, American Psychological Association. This paper is not the copy of record and may not exactly replicate the final, authoritative version of the article. Please do not copy or cite without authors permission. The final article will be available, upon publication, via its DOI: $10.1037 / \operatorname{dev0000467}$

Mother-Child Positivity and Negativity: Family-Wide and Child-Specific Main Effects and Interactions Predict Child Adjustment

Bonamy R. Olivera \& Alison Pike ${ }^{b}$

a Department of Psychology, Goldsmiths, University of London, UK

b School of Psychology, University of Sussex, UK

Correspondence to: Dr Bonamy R. Oliver (b.oliver@gold.ac.uk)

Department of Psychology, Goldsmiths, University of London, SE14 6NW

Tel: +44(0)2079197594

Acknowledgements

We are extremely grateful to all the families who took part in this study, the midwives for their help in recruiting them, and the ALSPAC team, which includes interviewers, computer and laboratory technicians, clerical workers, research scientists, volunteers, managers, receptionists, and nurses. The UK Medical Research Council, the Wellcome Trust (Ref: 102215/2/13/2) and the University of Bristol currently provide core support for ALSPAC. 


\begin{abstract}
Links between positive and negative aspects of the parent-child relationship and child adjustment are undisputed. Scholars recognize the importance of parental differential treatment (PDT) of siblings, yet, less is known about PDT in the context of the shared (family-wide) parent-child relationship climate, or about the extent to which positivity may buffer children's adjustment from negativity. Controlling for behavioral stability, we examined the potential for positive and negative parent-child processes to interact across and between child-specific and family-wide levels in the prediction of children's adjustment. Specifically, in a sample of 2039 UK families, we used multilevel models to examine child-specific and family-wide mother-child relationships (at 4 years) - including interactive processes -- in the prediction of prosocial behavior and conduct problems (at 7 years). The majority of variance in children's adjustment resided within-families: siblings were strikingly different. Accounting for behavioral stability, familywide negativity and negative PDT associated with both prosociality and conduct problems. Importantly, we demonstrated interactions between, i) family-wide negativity and negative PDT for conduct problems, as well as, ii) positive and negative PDT in the prediction of both prosocial behavior and conduct problems. Results suggest negative PDT associates with increased conduct problems over time, even when the overall family climate is low in negativity. They also indicate a buffering role of positive PDT on the deleterious effects of negative PDT for children's adjustment. Implications for both research and practice are discussed, including the importance of information gained by considering more than one child in the family.
\end{abstract}


Keywords: Parenting; prosocial behavior; conduct problems; multilevel modeling; Avon Longitudinal Study of Parents and Children (ALSPAC) 


\section{Mother-Child Positivity and Negativity: Family-Wide and Child-Specific Main Effects and Interactions Predict Child Adjustment}

Decades of research demonstrate that parent-child relationships are critical for children's socio-emotional adjustment (Maccoby, 2015), but most research uses one child per family (Plomin \& Daniels, 1987). Increasing recognition that parent-child relationships can vary within families in ways important for children's development (Browne, Plamondon, Prime, PuenteDuran, \& Wade, 2015; Jenkins, McGowan, \& Knafo-Noam, 2016; Oliver, 2015) motivated the present study. The current research contributes to the existing literature in three ways: 1) family-wide and child-specific parent-child relationships are considered simultaneously; 2) interaction effects between parent-child positivity and negativity are considered alongside their main effects; 3 ) both conduct problems and prosocial behavior are examined.

Prosocial behavior -- behaviors intended to benefit others, including helping, sharing and kind behaviors -- and conduct problems -- a range of childhood oppositional, disruptive and antisocial behaviors -- are key factors in children's behavioral development. Prosocial behaviors predict socioemotional competence and friendships, as well as long-term education, employment, and mental health outcomes (Jones, Greenberg, \& Crowley, 2015). On the other hand, conduct problems confer substantial risk for short- and long-term psychological, social, and academic difficulties for the individual, as well as associated societal burden (Fergusson, Horwood \& Ridder, 2005; Kim-Cohen, Caspi, Moffitt, Harrington, Milne, \& Poulton, 2003; Parsonage, Khan, \& Saunders, 2014). The transition from early- to middle-childhood is key for the development of both 
prosocial behavior and conduct problems because it is a time of considerable change, not least due to children's broadening socialization partners. Yet family influences remain critical: It is a period where, unlike adolescence, non-school time remains overwhelmingly in the context of the family. This, coupled with increasing child cognitive abilities and autonomy, transforms the nature of parent-child relationships, as children become more active partners, with a more detailed understanding of parents' perspectives (e.g., Reich \& Vandell, 2010).

\section{Parenting and Child Adjustment}

Parents' role in children's psychological development throughout infancy, childhood, and beyond, is irrefutable (Maccoby, 2015). Dimensions of parentchild relationships, commonly conceptualized as positivity (e.g., warmth, responsiveness, support) and negativity (e.g., hostility, criticism, harshness), are well-documented correlates of children's adjustment. For example, positivity associates with increased behavior regulation and social aptitude (e.g., Guajardo, Snyder, \& Petersen, 2009; Rutter, 1979), while negativity precipitates adjustment difficulties such as disruptive behavior and emotional distress (e.g., Amato \& Fowler, 2002; Pinquart, 2017). Moreover, a lack of positivity has been related to child externalizing problems (e.g., Eisenberg et al., 2005) and negativity to a lack of prosocial behavior (e.g., Knafo \& Plomin, 2006).

\section{Positivity and Negativity: Potential Interacting Processes}

Parent-child positivity and negativity are not opposite ends of a single continuum (Oliver, Trzaskowski, \& Plomin, 2014; Pettit, Bates, \& Dodge, 1997), and may have distinct roles for children's adjustment (Pettit, et al., 1997). Further, evidence suggests that parental positivity and negativity may interact in their prediction of child outcomes. Predicated on Baumrind's (1973) early work, 
the role of parental positivity or warmth in mitigating the adverse effects of negative parental control or discipline has been of particular interest. For example, maternal warmth and sensitivity have been shown to ameliorate links between parental physical/harsh discipline and child externalizing problems (e.g., Alink et al., 2009; Deater-Deckard, Ivy, \& Petrill, 2006). However, much less is known about these interactive processes for child prosocial behavior. We are aware of just one study of 139 Guyanese mothers that explicitly tested for an interaction (between maternal warmth and harshness), but no moderation was detected (Roopnarine, Jin \& Krishnakumar, 2014).

\section{Family-Wide and Child-Specific Effects}

The majority of research has used one child per family to demonstrate links between parent-child relationships and child behavioral outcomes. These studies yield a single parent-child relationship score which confounds two pieces of information - a parent's general parenting propensity, and that parent's unique relationship with the target child. For example, on a scale of 1-10, three motherchild dyads in different families could score 8 for mother-child positivity. In one family, this high mother-child positivity could be in the context of a mothersibling positivity score of 6 , indicating that the target child is not only receiving high positivity in relation to the population, but is also receiving more positivity than his/her sibling. In the second family, this high mother-child positivity could be in the context of a mother-sibling positivity score of 10 , indicating that although the target child is receiving high positivity in relation to the population, $\mathrm{s} / \mathrm{he}$ is receiving less positivity than his/her sibling. In the third family, a mothersibling positivity score of 8 could indicate a context of equal maternal positivity across child and sibling within the family. The experiences of the children -- and 
their mothers -- in these three families are very different, a nuance that may be important for child and sibling adjustment, and that is not captured in typical one-child-per-family research.

There is increasing recognition that siblings raised together develop surprisingly differently to one another, demonstrating that child-specific experiences are worthy of research attention (Plomin, 2011). In their seminal work, Daniels \& Plomin (1985) encouraged the examination of child-specific environmental factors for predicting children's adjustment, including measures of parenting that capture parents' differential treatment of children within families.

The past three decades have yielded consistent evidence that parental differential treatment (PDT) relates to child adjustment (e.g., Plomin, 2011) using a range of study designs and methods of assessing PDT. In most cases PDT is operationalized as a simple difference score (parenting towards one child subtracted from parenting towards his/her sibling), however PDT has also been measured by asking directly about experiences of favoritism. For example, young children's own reports of PDT using the difference score method related crosssectionally to parent reports of behavior problems (Coldwell, Pike, \& Dunn, 2008), mirroring earlier observational studies that also used the difference score method (e.g., Brody, Stoneman, \& McCoy, 1992; Dunn, Stocker, \& Plomin, 1990). In longitudinal analysis, maternal differential treatment has been shown to predict child adjustment over time using maternal reports of favoritism via a researcher-coded semi-structured interview (McGuire, Dunn, \& Plomin, 1995), via adolescents' reports of favoritism (Richmond, Stocker, \& Rienks, 2005), and via the difference score method using parent reports (Oliver, 2015). 
As illustrated above, the typical conceptualization is that differential sibling experiences, and specifically PDT, lead to differences in siblings' behavioral outcomes. However, evidence also shows that differences in siblings' traits (e.g., behavior or temperament) may elicit differences in parental treatment (AtzabaPoria \& Pike, 2008; Avinun \& Knafo, 2014; Jenkins, Rasbash, \& O’Connor, 2003). The bidirectional nature of parenting and child behavior has thus been demonstrated when considering multiple children within families.

The current study focuses on the traditional conceptualization: PDT predicting child behavior. In order to rationalize this interpretation, we use a longitudinal design to predict children's behavior from parent-child relationships (including PDT) at an earlier time point, while controlling for concurrent child behavior. The potential discrete roles of parent-child positivity and negativity at family-wide and child-specific levels are of key interest. In recent years multi-level modeling (MLM) has augmented the tools available to researchers, making it possible to explore both family-wide and child-specific effects within the same context (Jenkins et al., 2009).

\section{Multilevel Studies}

Using cross-sectional data from the National Longitudinal Survey of Children and Youth (NLSCY), Romano and colleagues (2005) investigated parenting in relation to aggression and prosocial behavior. To assess family-wide parenting, maternal reports of parenting towards all of her children were averaged. Children in families with higher average levels of hostile and/or punitive parenting displayed more aggression. In addition, children in families with lower average levels of parental positivity and higher average levels of punitive parenting engaged in less prosociality. To assess child-specific 
parenting, a discrepancy score was created for each child (i.e., the parenting score for the specific child subtracted from the family-average parenting score). These scores indicate whether the child is the recipient of more or less (e.g., hostile) parenting than his/her siblings. At this child-specific level, children experiencing greater than the family average maternal hostility were more aggressive and less prosocial, however experiencing more positivity was not predictive of child adjustment. Thus, both family-wide and child-specific negative parenting were key predictors of aggression and prosociality; in contrast, positive parenting was only important at the family-wide level, and only for prosocial behavior.

More recently, aggression and social relationship problems were examined in 397 families with preschool children using MLM (Meunier, Boyle, O’Connor \& Jenkins, 2013). Cumulative contextual risk associations with child adjustment were partially mediated through negative and positive PDT. Importantly, for social relationships, PDT was influential for all children in the family, regardless of which child was favored, critically suggesting family-wide as well as childspecific effects of PDT. That is, not only did disfavored children have more social relationship problems than their siblings, but additionally, in families with high levels of PDT, all children were more likely - compared with families with lower levels of PDT -- to have social relationship difficulties. In contrast, for aggression, PDT effects were aggravated for the disfavoured child. The authors emphasize the cross-sectional nature of their study, and posit that, while their findings suggest PDT may have different effects on different child outcomes, some child characteristics may increase PDT overall, while others (e.g., child aggression) may work to target parental disfavour towards a specific child. 
Existing MLM studies, then, suggest a role for positive and negative PDT in children's adjustment, asking related questions in different ways. Notably, no study has included a longitudinal element to examine parent-child relationship effects controlling for behavioral stability. Moreover, to our knowledge, potential interacting positive and negative parent-child processes have not been examined in relation to PDT, and not with MLM.

\section{Current Study}

Family systems theory (Minuchin, 1988) emphasises the inter-dependence of relationships within families, providing a formal framework for conceptualizing the dynamics whereby a parent's relationship with one child can have multiple meanings dependent on that parent's relationship with the child's sibling. From these and other theoretical frames (e.g., ideals of equity from social exchange theory; Huston \& Burgess 1979), the importance of considering differential treatment of siblings by their parents is apparent.

Yet, it is rare for PDT to account for more than a small proportion of variance in children's adjustment (Turkheimer \& Waldron, 2000). Critically, it is likely that experiences that differ between siblings in ways important for child adjustment are manifold, explaining small amounts of variance apiece (Plomin, DeFries, Knopik, \& Neiderhiser, 2016). We propose further that these main effects may be qualified by interactions with other aspects of family-wide and child-specific parent-child relations. We theorized two main interactive processes likely to be at play. First, we considered the overall parenting climate to be an important context for PDT (Tamrouti-Makkink, Dubas, Gerris, \& van Aken. 2004), considering both positive and negative aspects of parent-child relationships. Cross-sectional results in adolescent samples have demonstrated 
an overall parenting environment characterized by high conflict and/or low intimacy to exacerbate the associations between PDT and behavioural adjustment including externalizing problems and delinquency (e.g., Feinberg \& Hetherington, 2001). In contrast, however, in a longitudinal sample of children aged 3-5 years, Meunier and colleagues (2011) found associations between negative PDT and increasing externalizing problems over a two-year period to be exacerbated in the context of a more supportive, less negative overall parenting climate. These mixed and limited previous findings encouraged us to remain open to the direction of results we expected. Second, family research consistently demonstrates largely independent -- and potentially interacting roles -- for negative and positive aspects of parent-child relationships (Alink et al., 2009; Deater-Deckard, Ivy, \& Petrill, 2006; Pettit, et al., 1997). We believe the current study is unique in exploring potential interactions between parental positivity and negativity at within- and between-family levels of effect for child adjustment. We tested these moderation effects at all levels, but hypothesized that child-specific levels would be particularly important here. We theorized that a parent-child relationship characterized -- relative to a sibling -- by a lack of parent-child positivity and high parent-child negativity would be one likely to be particularly toxic for child outcomes.

We capitalized on the power afforded by a large, longitudinal sample to examine positive and negative aspects of parent-child relationships and their interactions, within an MLM framework. To our knowledge, this is the first longitudinal MLM study to account for behavioral stability in the examination of negative and positive PDT for prosocial behavior and conduct problems. Most important, we believe the current study is unique in exploring potential 
interactions between parental positivity and negativity at within- and betweenfamily levels of effect for child adjustment. We anticipated that a) the majority of the variance in adjustment would lie within-families, b) family-wide motherchild relationship positivity and negativity, and negative and positive PDT would explain variance in adjustment, c) positivity and negativity would interact in association with child outcomes such that, at least to some extent, parental positivity would buffer children from the deleterious effects of parental negativity.

\section{Method}

\section{Sample and Procedure}

ALSPAC (http://www.bristol.ac.uk/alspac/researchers/dataaccess/data-dictionary) is an ongoing population-based study designed to investigate the effects of a wide range of factors on children's health and development. All women resident in Avon, UK with expected dates of delivery between April 1, 1991 and December 31, 1992 were contacted and eligible for participation. The study cohort consisted of 14,541 pregnancies and 13,988 children still alive at 12 months of age. The sample is described in detail elsewhere (Boyd et al., 2013; Copeland, Shanahan, Costello, \& Angold, 2009). Ethical approval was obtained from the ALSPAC Law and Ethics Committee (IRB 00003312) and local Research Ethics Committees (Bristol and Weston, Southmead, and Frenchay Health Authorities).

By child age 4, questionnaires were sent to 12,349 mothers, and returned by 9,501 (76.9\%); at child age 7, questionnaires were sent to 10,662 mothers, of whom 8,505 (79.8\%) completed. ALSPAC data were available for the original target child and one older sibling only; to control as many extraneous factors as 
possible - and to maximize reliable identification of sibling data -- we elected to include the 2573 ALSPAC families for whom there were no other siblings. Of these, we excluded 530 families with an age-gap of more than 5 years, and four families with twins. The final sample thus consisted of 2039 families with two children. Comparing mothers in the selected two-sibling sample with those in the remaining (unselected) sample for whom 4-year data were available shows that the former were significantly (but inconsequentially) older $\left(M_{\text {selected }}=29.42\right.$ years, $M_{\text {unselected }}=28.45$ years; $\left.t(9497)=8.25, p<.001\right)$, and less likely to be single at the time of birth of the target child (Selected: $11.7 \%$, Unselected: $23.1 \%$; $\left.\chi^{2}=132.04, p<.001\right)$. These differences were expected since, for $57.7 \%$ of the unselected sample, the target child was the first (or only) born. Importantly, mothers in the selected and unselected samples were equally likely to selfidentify their ethnicity as non-white (Selected: 1.7\%, Unselected: $1.4 \% ; \chi^{2}=$ $132.04, p<.001$ ) and to have educational qualifications at A-level or higher, the national educational qualification taken at age 18 years in the United Kingdom (Selected: $39.6 \%$, Unselected: $37.4 \% ; \chi^{2}=1.13, p=.288$ ).

\section{Measures}

All measures were collected through postal questionnaire when the younger sibling was age 4 years (Time 1). Child adjustment measures were collected again when the younger sibling was 7 years (Time 2).

Child Adjustment was assessed using the Strengths and Difficulties Questionnaire (SDQ; Goodman, 1997), a widely used instrument with wellestablished reliability and validity, including comparisons between parent and teacher ratings for validation purposes (Goodman, 1997; Goodman, 2001). Statements about child behaviors over the previous six months were rated by 
mothers as 'not true' (coded 0), 'somewhat true' (1) or 'certainly true' (2). Two of five available subscales were included in the current study: prosocial behavior (five items, e.g., "considerate of other people's feelings"; $\alpha=.70-.75$ ), and conduct problems (five items, e.g., "often fights with other children or bullies them"; $\alpha=$ .49-.58). The internal consistency for conduct problems was moderate, consistent with other population-based studies using the scale (e.g., Jaffee, Hanscombe, Haworth, Davis \& Plomin, 2012). Indeed, variable internal consistency in scales is common when they comprise few items aiming assess diverse behaviors of a construct, since a low alpha simply indicates that the items are measuring different aspects.

Mother-child relationship was measured using_previously published scales (Dunn, Deater-Deckard, Pickering, \& O'Connor, 1998). With high face validity and previously demonstrated predictive validity (e.g., Dunn et al., 1998), these scales include indicators identified in the literature as aspects of parentchild relationships important for children's development (e.g., warmth, enjoyment, a lack of acceptance and rejection). Mother-child positivity was derived from four mother-report items, rated 'yes' (coded 2), 'sometimes' (1) or 'no' (0): "I really love this child", "this child makes me pretty happy", "this child is very affectionate to me", and "I feel very close to this child" ( $\alpha=.53-.63)$. Motherchild negativity was derived from four mother-report items, rated 'yes' (coded 2), 'sometimes' (1) or 'no' (0): “I often get very irritated with this child”, "I dislike the mess and noise that surrounds this child" "I have frequent battles of will with this child", and "this child gets on my nerves" (.63-.64).

Family-wide variables were calculated respectively as the average of mother-child positivity and negativity reported across the two siblings. Child- 
specific departures from these averages of mother-child positivity (positive PDT) and negativity (negative PDT) were then computed to index child-specific mother-child relationships that contribute to sibling differences in outcomes (Jenkins et al., 2009). For example, if one sibling in a family scores 6 and the other scores 2 for mother-child positivity, family-wide positivity would be $(6+2) / 2=4$. Subsequently, positive PDT for the first sibling would be $(6-4)=2$ and for the second sibling (2-4)=-2. Thus, a high positive PDT score indicates that the positivity in the mother-child relationship was more than the family average (i.e., more than their sibling); similarly, a high negative PDT score indicates that the negativity in the mother-child relationship was more than the family average (i.e., more than their sibling). We then examined all interaction processes with the potential to contribute both to sibling similarity (i.e., family-wide motherchild positivity*family-wide mother-child negativity), and to sibling differences in adjustment (i.e., family-wide mother-child positivity*positive PDT, familywide mother-child positivity*negative PDT, family-wide mother-child negativity*positive PDT, family-wide mother-child negativity*negative PDT, and

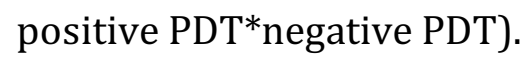

We were interested in establishing longitudinal prediction of children's adjustment at Time 2 from mother-child relationship variables at Time 1, over and above within-domain adjustment stability from Time 1 to Time 2 in order to account, in part, for evocative child effects. That is, we examined mother-child relationship variables at Time 1 prediction of prosocial behavior at Time 2, accounting for prosocial behavior at Time 1, and prediction of conduct problems at Time 2 accounting for conduct problems at Time 1 . To establish this stability at family-wide and child-specific levels, we calculated family-wide prosocial 
behavior (or conduct problems) at Time 1 as the family average of prosocial behavior (conduct problems) across siblings, and child-specific (differential) prosocial behavior (conduct problems) at Time 1 as departures from these averages.

\section{Analyses}

MLM offers the analysis of child-specific as well as family-wide effects that may be pertinent for child outcomes. MLM partitions between- and withingroup (here, "family") variance, and allows the inclusion of predictor variables to account for portions of these variances. Here, we are interested in family-wide factors that contribute to the extent to which siblings are similar to each other (in adjustment), and differentiated from children in other families, and childspecific factors that contribute to sibling differences in adjustment. Child-specific and family-wide variables are added as predictor variables to examine their potential contribution to the within- and between-family variance in child adjustment. In brief, MLM yields fixed effects (similar to traditional regression coefficients) as well as random effects, which represent the within- and betweenfamily level variance estimates once predictor variables are accounted for. Within-family variance also captures measurement error. The use of MLM for family data is described in more detail elsewhere (Jenkins et al., 2009).

Two identical sets of MLM were run with prosocial and conduct problems at Time 2 as dependent variables, and with child-specific and family-wide variables at Time 1 as independent predictors.

The first stage model (Model 1), yielded intraclass correlations (ICCs) - a measure of sibling similarity in adjustment outcomes. Time 1 domain-specific 
adjustment at both family-wide and child-specific levels was included in Model 2 to index behavioral stability. To assess the prediction of child adjustment after accounting for stability, subsequent models retained these stability indicators. Model 3 included all Time 1 child-specific ('within-family effects': child sex, differential within-domain adjustment, positive and negative PDT), and familywide ('between family effects': average within-domain adjustment, and familywide positivity and negativity) variables, but not their interactions. Finally, Model 4 included all predictors from Model 3, as well as interactions at childspecific (family-wide mother-child positivity*positive PDT, family-wide motherchild positivity*negative PDT, family-wide mother-child negativity*positive PDT, family-wide mother-child negativity*negative PDT, and positive PDT*negative PDT) and family-wide levels (family-wide positivity*family-wide negativity).

MLM models were estimated using Full Maximum Likelihood, and run using MPlus v.6 (Muthen \& Muthen, 1998-2010). All variables were centred to minimize collinearity using group-mean and grand-mean centring at levels 1 and 2 respectively, as recommended (e.g., Bauer \& Curran, 2005).

\section{Results}

\section{Preliminaries}

Descriptive statistics for all measures are given in Table 1.

Paired-samples $t$-tests revealed mean differences between siblings: at Time 1 older siblings showed more prosociality $(t(2019)=18.78, p<.001)$ and 
fewer conduct problems $(t(2019)=-8.49, p<.001)$; in contrast, younger siblings demonstrated more prosociality at Time $2(t(1592)=-3.22, p=.001)$, and no differences were found for conduct problems $(t(1593)=-0.98, p=.329)$. At Time 1, mothers reported more positivity in the relationship with younger than older siblings $(t(2011)=-7.88, p<.001)$, but similar levels of negativity $(t(2006)=-1.76$, $p=.079)$. Sibling correlations indicated substantial differential sibling behavior and mother-child relationships.

Correlations among study variables (also Table 1) were in expected directions and significant at $p<.001$, indicating substantial within-domain adjustment stability over time, and cross-domain associations. Mother-child positivity and negativity showed weak to strong correlations with each other and with child adjustment; associations between mother-child negativity and child conduct problems were particularly strong.

\section{MLM}

MLM results are presented in Table 2.

----Table 2----

Prosocial Behavior. As described in Analyses, Model 1 provided estimates of between- and within-family variance; the ICC (calculated as the between-family variance divided by the total variance) indicates sibling similarity for adjustment at Time 2. Reflecting the simple correlations (Table 1), for prosocial behavior, the ICC was $0.747 /(0.747+2.476)=.23$, suggesting some sibling similarity (23\%), but that the majority of variance resided within 
families. Model 2 indicated substantial behavioral stability at the family-wide level, calculated as the reduction in between-family variance from Model 1 to Model 2, divided by the total between-family variance, that is (0.747$0.257) / 0.747=65.6 \%$ of the between-family variance was explained. Childspecific stability was also seen, explaining $14.5 \%$ of the within-family variance in prosocial behavior, similarly calculated as the reduction in within-family variance from Model 1 to Model 2 (2.476-2.116) divided by the total withinfamily variance (2.476). Accounting for this behavioral stability, Model 3 indicated that changes in prosocial behavior over time were significantly predicted by child sex (girls showed more increased prosociality than boys), positive PDT (children with a mother-child relationship that was more positive than their sibling showed increases in prosocial behavior over time), negative PDT, (children with a mother-child relationship that was more negative than their sibling showed decreases in prosocial behavior over time), as well as family-wide mother-child relationship positivity and negativity (in expected directions). These variables accounted for a small amount (3.2\%) of supplementary variance in prosocial behavior at the family-wide level (the reduction in between-family variance from Model 2 to Model 3 (0.257-0.233), divided by the total between-family variance in Model 1 (0.747)). The reduction in within-family variance from Model 2 to Model 3 (2.116-2.039), divided by the total within-family variance in Model 1 (2.476) indicated that $3.1 \%$ additional within-family level variance was explained.

The final model (Model 4), estimated the prediction of prosociality afforded by interactions between our mother-child relationship variables accounting for all main effects previously described. Negligible additional 
variance was explained (within-family: reduction in variance from Model 3 to Model 4 (2.039-2.036) divided by total within-family variance in Model 1 $(2.476)=0.1 \%$; between-family: reduction in variance from Model 3 to Model 4 (0.233-0.227) divided by total between-family variance in Model $1(0.747)=$ $.08 \%)$. Thus, the value in the interaction findings was not in explaining additional variance, but rather in uncovering the more nuanced family processes potentially masked by the main effects.

Using simple slopes analyses to illustrate this interaction ${ }^{1}$ (see Figure 1a), we see a buffering role of positive PDT: the inverse relationship between negative PDT and prosocial behavior was stronger when positive PDT was lower $(-1 \mathrm{SD}: \beta=-.12, t=-4.08, p<.001 ; M: \beta=-.07, t=-3.16, p=.002 ;+1 S D: \beta=-.03, t$ $=-0.95, p=.342$ ). Our interpretation of this is that, while children with a less negative mother-child relationship than their sibling presented higher levels of prosocial behavior regardless of the child-specific mother-child positivity, for children with a mother-child relationship that was more negative than their sibling, positive PDT played an important role, protecting their prosocial development from the deleterious effects of negative PDT.

----Figure 1----

To further interpret this interaction, we examined regions of significance using the Johnson-Neyman procedure (Johnson \& Neyman, 1936). This procedure is used to identify the point(s) along a continuous moderator (here,

\footnotetext{
${ }^{1}$ Simple slopes are used for illustration purposes only, and should not be used to conclude the size of interaction effects (Aguinis, Gottfredson, \& Culpepper, 2013)
} 
average negativity or positive PDT) at which the association between the independent variable (negative PDT) and dependent variable (child adjustment) transitions from/to statistical significance. Figure 1 b depicts the JohnsonNeyman regions of significance for the moderating effect of positive PDT on the association between negative PDT and child prosocial behaviour. The regions of significance can be identified as those where the 95\% Upper (U) and Lower (L) confidence intervals $(\mathrm{CI})$ do not overlap 0 on the $\mathrm{Y}$-axis. Note that 0 on the $\mathrm{X}$-axis (PDT) indicates that the two siblings have mother-child relationships reported to be similar to each other. A score above 0 refers to children with a mother-child relationship that was reported to be more positive than their sibling, and a score below 0 refers to children with reportedly less positivity in the mother-child relationship than their sibling. The axis refers to SD differences. There were two regions of significance for this interaction: i) where positive PDT was below 0.21SD, and, ii) where positive PDT was above 2.36SD. With regards i), 96.3\% of children lay in this significance region, where the mother-child relationship was equally or less positive than that of their sibling. This sample portion was large, since it included the majority of children ( $93 \%$ of our sample) whose mothers reported equal positivity in the relationship with each of her children (i.e., positive PDT was at $0^{2}$ ). As depicted in Figure $1 \mathrm{a}$, the association between negative PDT and prosocial behavior was significant here. For the $3.5 \%$ of children for whom positivity was $>=0.21$ SD but $<=2.36$ SD more than their sibling, the association between negative PDT and prosociality was not significant. We interpret these results as implying a buffering effect of positive

\footnotetext{
${ }^{2}$ Note that mothers were more discriminating in reporting their negative PDT which was at 0 for $44 \%$ of the sample.
} 
PDT on negative PDT for prosocial behaviour, since, only when positive PDT was high (i.e., for children with a mother-child relationship that was more positive than their sibling), was the association between negative PDT not significantly associated with changes in prosocial behaviour over time. Regarding ii), positive PDT was 2.36SD or higher, and the association between negative PDT and prosocial behavior was again significant. However, we note that this region involved $0.2 \%$ of children (just four families). As such, we consider these cases to be outliers, and do not interpret this further.

Conduct Problems. For conduct problems, MLM results are also given in Table 2. The ICC (Model 1) indicated that $0.458 /(0.458+1.523)=23 \%$ of the variance in conduct problems resided between families, but the majority lay within families. Similar to results for prosocial behavior, comparing Model 1 and Model 2 indicated substantial behavioral stability. That is, behavioral stability explained $69.7 \%$ of the between-family variance (calculated as the reduction in between-family variance from Model $1(0.458)$ to Model $2(0.139)$ divided by the total between-family variance (0.458)), and $14.2 \%$ of the within-family variance (the reduction in within-family variance from Model 1 (1.523) to Model 2 (1.307) divided by the within-family variance from Model 1 (1.523)). Over and above this stability, Model 3 indicated a role for child-specific (sex, positive PDT, and negative PDT) as well as family-wide (average mother-child positivity and negativity) predictors of change in conduct problems, all with effects in the opposite direction from those found for prosocial behavior, as expected. These variables accounted for an additional 3.1\% of the between-family variance (0.139 (within-family variance Model 2) - 0.125 (Model 3))/0.458 (Model 1)) and $1.5 \%$ of the within-family variance (1.307 (Model 2) - 1.284 (Model 3)/1.523 
(Model 1)).

Finally, Model 4 indicated the prediction of conduct problems from our interaction variables accounting for all main effects. As before, this model showed little additional variance explained (Within-family: 1.284 (Model 3) $1.275($ Model 4)/1.523 (Model 1) = 0.6\%; Between-family: 0.125 (Model 3) $0.125($ Model 4)/0.458 (Model 1) = 0\%).

Here, two important interactions were revealed. First, a significant interaction was found between average mother-child negativity and negative PDT. Probing this with simple slopes for illustration purposes (see Figure 2a) indicated a pattern of effect that was stronger when family-wide mother-child negativity was lower (-1SD: $\beta=.16, t=2.69, p=.007 ; M: \beta=.08, t=2.60, p=$ $.010 ;+1 S D: \beta=.00, t=0.11, p=.915)$. In families where average mother-child negativity was high, conduct problems increased over the study period, but negative PDT made little difference. However, in families where average motherchild negativity was low, children with a mother-child relationship that was more negative than their sibling were more likely to show increasing conduct problems.

\section{----Figure 2----}

The Johnson-Neyman regions of significance for the moderating effect of average mother-child negativity on the association between negative PDT and child conduct problems are shown in Figure $2 \mathrm{~b}$. For children for whom the family-wide mother-child relationship negativity was below $0.63 \mathrm{SD}$ (70.5\% of children), the association between negative PDT and conduct problems was significant, whereas above this value the association was not significant. We 
interpret this as suggesting that, in families where mother-child negativity is, overall, low, differential mother-child negativity may be particularly pertinent for increasing conduct problems.

Second, mirroring the results for prosocial behavior, the interaction between negative and positive PDT was also significant for conduct problems. Using simple slopes analyses to illustrate this interaction (see Figure 3a), the buffering role of mother-child positive PDT was again apparent. Negative PDT and conduct problems were most rigorously associated when positive PDT was lower (-1SD: $\beta=.10, t=3.80, p<.001 ; \mathrm{M}: \beta=.06, \mathrm{t}=2.90, \mathrm{p}=.004 ;+1 \mathrm{SD}: \beta=.02$, $\mathrm{t}=0.82, \mathrm{p}=.414$ ). Here, children with a more positive mother-child relationship than their sibling presented with lower levels of conduct problems regardless of child-specific negativity, whereas, for children with a less positive mother-child relationship than their sibling, negative PDT was significantly associated with increasing conduct problems in middle childhood.

\section{----Figure 3----}

The J-N regions of significance for the interaction between positive and negative PDT in association with conduct problems (Figure 3b) demonstrated that the vast majority of children were in the significant region (including, as before, children for whom mothers reported no positive PDT). That is, for the $96.3 \%$ of children for whom the mother-child relationship was equally, or less positive than that of their sibling, the association between negative PDT and conduct problems was significant: the child with a less negative mother-child relationship had lower levels of later conduct problems. However, for the $3.7 \%$ 
of children who exceeded $0.17 \mathrm{SD}$ more positivity in their mother-child relationship than their sibling, negative PDT did not significantly associate with conduct problems. Echoing the results for prosocial behaviour, we intuit these findings as a buffering role for positive PDT.

\section{Supplementary Analyses}

Prosocial behavior and conduct problems were related, with effect sizes large, and essentially equivalent for within- and across-domain associations (Table 1). Thus, in post-hoc analyses, we repeated MLM models accounting for cross- as well as within-domain child-specific and family average behaviors at Time 1. The results remained unchanged. This is in line with studies demonstrating these aspects of children's socio-emotional adjustment to be far from two sides of the same coin (e.g., Krueger, Hicks, \& McGue, 2001). With our principal focus on interactive processes, and in the interests of simplicity, we elected to present the within-domain findings (cross-domain results available from first author).

\section{Discussion}

Within a multilevel sibling design, we examined mother-child positivity and negativity in relation to change in child prosocial behavior and conduct problems over a three-year period in middle childhood. Replicating previous findings, for both prosocial behavior and conduct problems, under a quarter of the variance in adjustment lay between families, with the majority residing within families (e.g., Plomin, 2011; Romano et al., 2005). Accounting for behavioral stability, in expected directions, family-wide mother-child negativity was related to both prosocial behavior and conduct problems (e.g., Amato \& Fowler, 2002; Knafo \& 
Plomin, 2006). Additionally, we uncovered PDT associations also with negative valence, in accord with existing research (Daniels \& Plomin, 1985; TamroutiMakkink et al., 2004).

The main effect of mother-child positivity was significant, as shown in Model 3, however, in our final model we demonstrate this main effect to be qualified by the interactions we observed. We posit this to suggest that variance explained by parental positivity may be additionally reflected in interactive, child-specific processes. We were particularly interested in harnessing the power afforded by our large sample to examine such interactive effects, potentially at both within- and between-family levels. We revealed that a stronger effect of negative PDT on increasing conduct problems was apparent in families where overall mother-child negativity was low, in contrast with crosssectional findings in adolescence (Feinberg \& Hetherington, 2001), but in line with findings in a longitudinal sample of young children (Meunier et al., 2011). We suggest that, for families characterized by high parental negativity overall, the salience of PDT over time for young children's adjustment maybe swamped by the detrimental effect of this ambient hostility. Our results demonstrate a more ideal context for children's behavioral development to be where the mother-child relationship climate is low in negativity and there is little PDT. Moreover, a buffering effect of positive PDT was found for associations between negative PDT and both adjustment domains. We discuss these results in terms of key themes to emerge, before acknowledging study strengths, limitations and suggested future directions, and implications of our findings. Adjustment stability lies within as well as between families

We evince behavioral stability over time that was similar across prosocial 
and conduct problem domains. Stability lay largely at the family-wide level, indicative of some families having children with consistently lower overall levels of prosocial behavior and higher overall levels of conduct problems than other families. Importantly, after accounting for these family-wide behavioral consistencies, the results also revealed behavioral constancy within-families, suggesting that child-specific presentations of behavior are also stable. In other words, the same child of the sibling pair is consistently less prosocial or shows consistently more conduct problems than their sibling.

Child-specific behavioral stability is likely due to genetic as well as environmental factors. In a wealth of studies, after accounting for genetics, environmental influences on children's adjustment are found to be considerable, particularly non-shared effects (NSE). However, the NSE component of variance includes measurement error, and as such these influences are commonly considered to be transient, providing time-limited explanations of variance (Lewis \& Plomin, 2015). Here, we show that a reasonable proportion of the total variance in adjustment was accounted for by non-shared parenting (PDT), even after accounting for behavioral stability. That is, while PDT may in part be due to differences in the characteristics of the children and is likely to reflect geneenvironment correlations (Knafo \& Jaffee, 2013), we theorize that PDT also reflects stability in parental differential attributions and attitudes towards children within a family, regardless of child behaviors. This is an important step in understanding family dynamics and the links between parent-child relationships and children's outcomes.

Positivity and Negativity are interactive processes 
One of our most interesting results was consistent across behavioral domains (and robust when accounting for cross-domains): negative and positive PDT interacted in their prediction of child adjustment. Perhaps not surprisingly, the children with the most favorable outcomes were those with less negativity and more positivity in the mother-child relationship than their sibling. Of note, however, is that this interaction suggested a buffering role of positive PDT on the deleterious effects of negative PDT for children's adjustment. For conduct problems, these findings converge with existing literature examining parental warmth and harsh discipline (e.g., Alink et al., 2009; Deater-Deckard et al., 2006). For prosocial behavior, previous findings are scarce, to our knowledge revealing no such moderation effects (Roopnarine et al., 2014). However, our results for prosociality largely mirror those for conduct problems, and it is likely that we were able to detect this interaction because of the large sample size and the inclusion of within- as well as between-family effects.

In this way, we extend the existing literatures, demonstrating the interaction to lie at child-specific levels, and unpacking the main effects of positivity. Although the additional variance explained by these interactive effects was negligible, we found considerable depletion of the prediction afforded by positivity alone. Our findings suggest that the role of positivity may be better understood as a modifier of the negativity bias so pervasive in interpersonal relationships and psychological phenomena (Baumeister, Bratslavsky, Finkenauer, \& Vohs, 2001). The negligible amount of variance explained by adding the interaction effects is also likely due to the small proportion of our families for whom marked positive PDT existed. The large sample allowed us to uncover commonly masked interactive processes for children's behavioural 
development that are in line with demonstrations that low but not high levels of PDT may be tolerated (Meunier, Bisceglia, \& Jenkins 2012). Further, we would argue that our sample and parent-report measures likely underestimate PDT experienced in families, and as such underplay the critical role it has for children's adjustment over time. Exploring regions of significance (rarely seen in the PDT literature) in the interpretation of interaction effects is key for understanding nuanced family processes that may be uncommon, but essential for children involved.

\section{Limitations and future directions}

Our study strengths include its longitudinal nature, the sample size, and the use of MLM, which, as we demonstrate, provides an important tool for examining within- and between- family effects, allowing advances in our understanding of family processes. However, we acknowledge some limitations. For example, our focus on families with only two children (due to data availability) potentially restricts generalizability. Indeed, to our knowledge, there is little research explicitly examining differences in the effects of PDT as a function of family size, and we encourage follow-on analyses in complex families. Of note, measurement error is captured by the within-family variance, and we acknowledge the variable internal consistency in our scales. The sole use of maternal reports here may inflate our associations due to rater bias and contrast effects (Saudino, 2004), as well as "perceiver effects" whereby parent characteristics affect their interpretation of child and own behaviors (Manders, Janssens, Cook, De Bruyn, \& Scholte, 2009). Previous studies indicate that although parent reports identify 
less PDT, the PDT reported by mothers is associated at least as strongly with child adjustment as is PDT reported by children (e.g., Coldwell, Pike, \& Dunn, 2008). In fact, maternally reported PDT may capture particularly detrimental differential treatment because parents report little PDT, and these reports run counter to social desirability biases. Studies examining more detailed mother reports, father reports, child reports and independent observations would all be of interest for further study.

Finally, it is possible that decreasing prosociality and increasing conduct problems between time periods in turn increased parental negativity (Meunier et al., 2013). These effects were masked in our models since we do not include mother-child assessments at Time 2 . Relevant data were available, however, the complexity of accounting for all potential interactions across all levels were beyond the scope of the study; we encourage scholars to consider these issues in future study designs.

\section{Implications and Conclusions}

A primary implication of the current findings is that increasing motherchild positivity, and reducing negativity, as well as decreasing PDT within families has the potential to promote optimal child outcomes during middle childhood. Further, the extent to which parent-child positivity may not only be a driver of behavioral change, but additionally plays a key moderating role for negativity is of interest. Our findings bolster the evidence base for family interventions that promote positivity in the parent-child relationship before addressing (negative) disciplinary strategies (e.g., Incredible Years (WebsterStratton \& Reid, 2010); Triple P (e.g., Sanders, Markie-Dadds \& Turner, 2003)), since the buffering role of positivity may be critical for behavioural change in the 
face of negativity. Subsequent increases in children's prosocial behavior and reductions in their conduct problems in turn are likely to reduce parent-child negativity.

We additionally highlight the practical importance of parental differential treatment: while overall positivity and negativity within the family relationship climate is important, key associations with behavior change were shown to involve child-specific mother-child relationships. With most children growing up with siblings, the large parent-child literature remains imbalanced in its majority focus on one-child, between-family effects. Further, our findings suggest that interventions may be minded -- in addition to their traditional aims -- to examine parent-child relationships in the context of those with siblings, and to reduce PDT explicitly. A child targeted for intervention will presumably be the child in the family also demonstrating the most difficult behavior, and we note the possibility of evocative child effects here; in turn, we speculate that there will be more PDT for these clinically referred families, since contextual risk has been shown to associate with increased PDT (Meunier et al., 2013), potentially leaving children vulnerable to the potent effects of negative PDT, unbuffered by positivity. While practitioners and policy-makers increasingly address multiple factors within the family, family dynamics involving siblings are largely neglected. We posit that due attention could be given to underlying interactive processes, as well as to multiple children in the family where appropriate, addressing PDT head-on. 


\section{References}

Alink, L. R., Mesman, J., Van Zeijl, J., Stolk, M. N., Juffer, F., Bakermans-Kranenburg, M. J., ... \& Koot, H. M. (2009). Maternal sensitivity moderates the relation between negative discipline and aggression in early childhood. Social Development, 18, 99-120. doi:10.1111/j.1467-9507.2008.00478.x

Aguinis, H., Gottfredson, R. K., \& Culpepper, S. A. (2013). Best practice recommendations for estimating interaction effects using multilevel modeling. Journal of Management, 39, 1490-1528.

Amato, P. R., \& Fowler, F. (2002). Parenting practices, child adjustment, and family diversity. Journal of Marriage and Family, 64, 703-716. doi: 10.1111/j.1741-3737.2002.00703.x

Atzaba-Poria, N. \& Pike, A. (2008). Correlates of parental differential treatment: Parental and contextual factors during middle childhood. Child Development, 79, 217-232.

Avinun, R. \& Knafo, A. (2014). Parenting as a reaction evoked by children's genotype: A meta-analysis of children-as-twins studies. Personality and Social Psychology Review, 18, 87-102.

Baumrind, D. (1973). The development of instrumental competence through socialization. In A. D. Pick (Ed.), Minnesota Symposia on Child Psychology (Vol. 7, pp. 3-46). Minneapolis: University of Minnesota Press.

Baumeister, R. F., Bratslavsky, E., Finkenauer, C., \& Vohs, K. D. (2001). Bad is stronger than good. Review of General Psychology, 5, 323. doi:10.1037/10892680.5.4.323

Boyd, A., Golding, J., Macleod, J., Lawlor, D. A., Fraser, A., Henderson, J. et al. (2013) Cohort profile: the 'Children of the 90s'-index offspring of the Avon 
Longitudinal Study of Parents and Children. International Journal of Epidemiology, 42, 111-127. doi: 10.1093/ije/dys064

Brody, G. H., Stoneman, Z., \& McCoy, J. K. (1992). Parental differential treatment of siblings and sibling differences in negative emotionality. Journal of Marriage and the Family, 54(3), 643-651. doi: 10.2307/353250

Browne, D. T., Plamondon, A., Prime, H., Puente-Duran, S., \& Wade, M. (2015). Cumulative risk and developmental health: An argument for the importance of a family-wide science. WIREs Cognitive Science, 6(4), 397-407. doi:10.1002/wcs.1349

Coldwell, J., Pike, A., \& Dunn, J. (2008). Maternal differential treatment and child adjustment: A multi-informant approach. Social Development, 17(3), 596612. doi: 10.1111/j.1467-9507.2007.00440.x

Copeland, W., Shanahan, L., Costello, E.J., \& Angold, A. (2009). Configurations of common childhood psychosocial risk factors. Journal of Child Psychology and Psychiatry, 50, 451-459. doi: 10.1111/j.1469-7610.2008.02005.x

Daniels, D., \& Plomin, R. (1985). Differential experience of siblings in the same family. Developmental Psychology, 21, 747-760. doi: 10.1037/00121649.21.5.747

Deater-Deckard, K., Ivy, L., \& Petrill, S. A. (2006). Maternal warmth moderates the link between physical punishment and child externalizing problems: A parent-offspring behavior genetic analysis. Parenting: Science and Practice, 6, 59-78. doi:10.1207/s15327922par0601_3

Dunn, J., Deater-Deckard, K., Pickering, K., O'Connor, T. G., \& Golding, J. (1998). Children's adjustment and prosocial behaviour in step-, single-parent, and non-stepfamily settings: Findings from a community study. Journal of Child 
Psychology and Psychiatry, 39(8), 1083-1095. doi: 10.1111/14697610.00413

Dunn, J., Stocker, C., \& Plomin, R. (1990). Nonshared experiences within the family: Correlates of behavioral problems in middle childhood. Development and Psychopathology, 2, 113-126. doi:10.1017/S0954579400000651

Eisenberg, N., Zhou, Q., Spinrad, T. L., Valiente, C., Fabes, R. A., \& Liew, J. (2005). Relations among positive parenting, children's effortful control, and externalizing problems: A three-wave longitudinal study. Child Development, 76, 1055-1071. doi:10.1111/j.1467-8624.2005.00897.x

Feinberg, M., \& Hetherington, E. M. (2001). Differential parenting as a withinfamily variable. Journal of Family Psychology, 15, 22-37. doi:10.1037/08933200.15.1.22

Fergusson, D. M., Horwood, J. L., \& Ridder, E. M. (2005). Show me the child at seven: the consequences of conduct problems in childhood for psychosocial functioning in adulthood. Journal of Child Psychology and Psychiatry, 46(8), 837-849

Goodman, R. (1997). The Strengths and Difficulties Questionnaire: A research note. Journal of Child Psychology and Psychiatry 38, 581-586. doi: 10.1111/j.1469-7610.1997.tb01545.x

Goodman, R. (2001). Psychometric properties of the Strengths and Difficulties Questionnaire (SDQ). Journal of the American Academy of Child and Adolescent Psychiatry, 40, 1337-1345. doi:10.1097/00004583-20011100000015

Guajardo, N. R., Snyder, G., \& Petersen, R. (2009). Relationships among parenting practices, parental stress, child behaviour, and children's social-cognitive 
development. Infant and Child Development, 18, 37-60. doi:

$10.1002 /$ icd.578

Huston, T. L., \& Burgess, R. L. (1979). Social exchange in developing relationships: An overview. In R. L. Burgess \& T. L. Huston (Eds.), Social exchange in developing relationships (pp. 3-28). New York: Academic Press.

Jaffee, S. R., Hanscombe, K. B., Haworth, C. M. A., Davis, O. S. P., \& Plomin, R. (2012). Chaotic homes and children's disruptive behavior: A longitudinal cross-lagged twin study. Psychological Science, 23(6), 643-650. doi: $10.1177 / 0956797611431693$

Jenkins J. M., Cheung C., Frampton K., Rasbash J., Boyle M. H., Georgiades K. (2009). The use of multilevel modeling for the investigation of family process. International Journal of Developmental Science, 3, 131-149. doi: 10.3233/DEV-2009-3204

Jenkins, J. M., McGowan, P., \& Knafo-Noam, A. (2016). Parent-offspring transaction: Mechanisms and the value of within family designs. Hormones and Behavior, 77, 53-61. doi: 10.1016/j.yhbeh.2015.06.018

Jenkins, J. M., Rasbash, J., \& O'Connor, T. G. (2003). The role of the shared family context in differential parenting. Developmental Psychology, 39(1), 99-113. doi: 10.1037/0012-1649.39.1.99

Johnson, P. O., \& Neyman, J. (1936). Tests of certain linear hypotheses and their application to some educational problems. Statistical Research Memoirs, 1, 57-93.

Jones, D. E., Greenberg, M., \& Crowley, M. (2015). Early social-emotional functioning and public health: The relationship between kindergarten social 
competence and future wellness. American Journal of Public Health, 105, 2283-2290. 10.2105/AJPH.2015.302630

Kim-Cohen, J., Caspi, A., Moffitt, T. E., Harrington, H., Milne, B. J., \& Poulton, R. (2003). Prior juvenile diagnoses in adults with mental disorder Developmental follow-back of a prospective-longitudinal cohort. Archives of General Psychiatry, 60(7), 709-717.

Knafo, A., \& Jaffee, S. R. (2013). Gene-environment correlation in developmental psychopathology. Developmental Psychopathology, 25, 1-6. doi:

\section{$10.1017 / S 0954579412000855$}

Knafo, A., \& Plomin, R. (2006). Parental discipline and affection and children's prosocial behavior: Genetic and environmental links. Journal of Personality and Social Psychology, 90, 147-164. doi: 10.1037/0022-3514.90.1.147

Krueger, R. F., Hicks, B. M., \& McGue, M. (2001). Altruism and antisocial behavior: Independent tendencies, unique personality correlates, distinct etiologies. Psychological Science, 12(5), 397-402. doi:10.1111/1467-9280.00373

Lewis, G. J., \& Plomin, R. (2015). Heritable influences on behavioural problems from early childhood to mid-adolescence: Evidence for genetic stability and innovation. Psychological Medicine, 45, 2171-2179. doi:10.1017/S0033291715000173

Manders, W. A., Janssens, J. M. A. M., Cook, W. L., Oud, J. H. L., De Bruyn, E. E. J., \& Scholte, R. H. J. (2009). Perceptions of Problem Behavior in Adolescents' Families: Perceiver, Target, and Family Effects. Journal of Youth and Adolescence, 38, 1328-1338. doi:10.1007/s10964-008-9339-y 
McGuire, S., Dunn, J., \& Plomin, R. (1995). Maternal differential treatment of siblings and children's behavioral problems: A longitudinal study. Development and Psychopathology, 7(3), 515-528.

Meunier, J. C., Bisceglia, R., \& Jenkins, J. M. (2012). Differential parenting and children's behavioral problems: Curvilinear associations and mother-father combined effects. Developmental Psychology, 48, 987-1002. doi:10.1037/a0026321

Meunier, J. C., Boyle, M., O'Connor, T. G., \& Jenkins, J. M. (2013). Multilevel mediation: Cumulative contextual risk, maternal differential treatment, and children's behavior within families. Child Development, 84, 1594-1615. doi: 10.1111/cdev.12066

Meunier, J. C., Roskam, I., Stievenart, M., van de Moortele, G., Browne, D. T., \& Kumar, A. (2011). Externalizing behavior trajectories: The role of parenting, sibling relationships and child personality. Journal of Applied Developmental Psychology, 32, 20-33. doi:10.1016/j.appdev.2010.09.006 Minuchin, P. (1988). Relationships within the family: A systems perspective on development. In R. Hinde \& J. Stevenson-Hinde (Eds.), Relationships within families: Mutual influences (pp. 7-26). Oxford, UK: Clarendon.

Oliver, B. R. (2015). Unpacking externalising problems: negative parenting associations for conduct problems and irritability. British Journal of Psychiatry (Open), 1, 42-47. doi:10.1192/bjpo.bp.115.000125

Oliver, B. R., Trzaskowski, M., \& Plomin, R. (2014). Genetics of parenting: The power of the dark side. Developmental Psychology, 50(4), 1233-1240. doi: $10.1037 / \mathrm{a} 0035388$ 
Parsonage, M., Khan, L., Saunders, A. Building a Better Future: the Lifetime Costs of Childhood Behavioural Problems and the Benefits of Early Intervention. Centre for Mental Health, London; 2014

Pettit, G. S., Bates, J. E., \& Dodge, K. A. (1997). Supportive parenting, ecological context, and children's adjustment: A seven-year longitudinal study. Child Development, 68, 908-923. doi:10.1111/j.1467-8624.1997.tb01970.x

Pinquart, M. (2017). Associations of parenting dimensions and styles with externalizing problems of children and adolescents: An updated metaanalysis. Developmental Psychology, 53(5), 873-932. doi:

$10.1037 / \operatorname{dev} 0000295$

Plomin, R. (2011). Commentary: Why are children in the same family so different? Non-shared environment three decades later. International Journal of Epidemiology, 40, 582-592. doi:10.1093/ije/dyq144

Plomin, R., \& Daniels, D. (1987). Why are children in the same family so different from one another? Behavioral and Brain Sciences, 10, 1 - 60.

Reich, S. M. \& Vandell, D. L. (2010). The interplay between parents and peers as socializing influences in children's development. In P. K. Smith \& C. H. Hart (Eds.), The Wiley-Blackwell handbook of childhood social development, $2^{\text {nd }}$ Edition. Oxford: Wiley-Blackwell. doi: 10.1002/9781444390933.ch14

Richmond, M. K., Stocker, C. M., \& Rienks, S. L. (2005). Longitudinal associations between sibling relationship quality, parental differential treatment, and children's adjustment. Journal of Family Psychology, 19(4), 550.

Romano, E., Tremblay, R. E., Boulerice, B., \& Swisher, R. (2005). Multilevel correlates of childhood physical aggression and prosocial behavior. Journal of Abnormal Child Psychology, 33, 565-578. doi:10.1007/s10802-005-6738-3 
Roopnarine, J. L., Jin, B., \& Krishnakumar, A. (2014). Do Guyanese mothers' levels of warmth moderate the association between harshness and justness of physical punishment and preschoolers' prosocial behaviours and anger?. International Journal of Psychology, 49, 271-279. doi:10.1002/ijop.12029

Rutter, M. (1979). Protective factors in children's responses to stress and disadvantage. Annals of the Academy of Medicine Singapore, 8, 324-338.

Sanders, M. R., Markie-Dadds, C., \& Turner, K. M. T. (2003). Theoretical, scientific and clinical foundations of the Triple P-Positive Parenting Program: A population approach to the promotion of parenting competence. Parenting Research and Practice Monograph, 1, 1-21.

Saudino K. J. (2004). Night and day: Are siblings as different in temperament as parents say they are? Journal of Personality and Social Psychology, 87, 698706. doi:10.1037/0022-3514.87.5.698

Tamrouti-Makkink, I. D., Dubas, J. S., Gerris, J. R. M., \& van Aken, M. A. G. (2004). The relation between the absolute level of parenting and differential parental treatment with adolescent siblings' adjustment. Journal of Child Psychology and Psychiatry, 45, 1397-1406. doi:10.1111/j.14697610.2004.00331.x

Turkheimer, E., \& Waldron, M. (2000). Nonshared environment: a theoretical, methodological, and quantitative review. Psychological Bulletin, 126, 78-108. doi:10.1037/0033-2909.126.1.78

Webster-Stratton, C., \& Reid, M. J. (2010). The Incredible Years parents, teachers, and children training series: A multifaceted treatment approach for young children with conduct disorders. Guilford Press. 
Table 1

Descriptive Statistics and Correlations Among Study Variables

\begin{tabular}{|c|c|c|c|c|c|c|c|c|}
\hline & \multicolumn{3}{|c|}{ Descriptives } & \multicolumn{5}{|c|}{ Correlations } \\
\hline & Older Sibling & Younger Sibling & Sibling & 1. & 2. & 3. & 4. & 5. \\
\hline & $\mathrm{M}(\mathrm{SD})$ & $\mathrm{M}(\mathrm{SD})$ & $r$ & & & & & \\
\hline \multicolumn{9}{|l|}{ Time 1} \\
\hline 1. Prosocial behavior ${ }^{\dagger}$ & $7.90(1.79)$ & $6.96(2.03)$ & $.30^{* * *}$ & -- & & & & \\
\hline 2. Conduct problems ${ }^{\dagger}$ & $1.64(1.42)$ & $1.96(1.37)$ & $.25^{* * *}$ & $-.42^{* * *}$ & -- & & & \\
\hline 3. Mother-child positivity ${ }^{\dagger}$ & $7.82(0.74)$ & $7.96(0.40)$ & $.21^{* * *}$ & $.13^{* * *}$ & $-.17^{* * *}$ & -- & & \\
\hline 4. Mother-child negativity & $2.44(2.45)$ & $2.53(2.41)$ & $.48^{* * *}$ & $-.29 * * *$ & $.51^{* * *}$ & $-.19^{* * *}$ & -- & \\
\hline Time 2 & & & & & & & & -- \\
\hline 5. Prosocial behavior ${ }^{\dagger}$ & $8.08(1.86)$ & $8.25(1.73)$ & $.24^{* * *}$ & $.48^{* * *}$ & $-.29 * * *$ & $.17^{* * *}$ & $-.24 * * *$ & \\
\hline 6. Conduct problems & $1.49(1.46)$ & $1.54(1.35)$ & $.23^{* * *}$ & $-.26^{* * *}$ & $.51^{* * *}$ & $-.17^{* * *}$ & $.36^{* * *}$ & $-.42^{* * *}$ \\
\hline
\end{tabular}


Table 2

Predicting Prosocial Behavior and Conduct Problems at Time 2: Fixed and Random Effects

Unstandardized Parameter Estimate (SE)
Prosocial Behavior
Conduct Problems

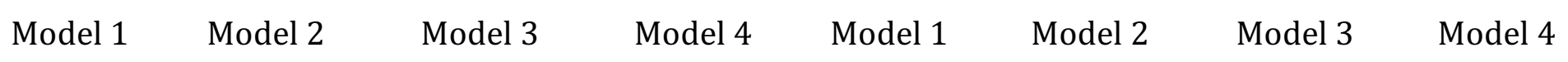

Fixed effects

Within-Family

Sex

CS Adjustment (Time 1)

Positive PDT

Negative PDT

Av. positivity*Positive PDT

$\begin{array}{ccc} & -.41(.05)^{* * *} & -.41(.05)^{* * *} \\ .37(.02)^{* * *} & .32(.02)^{* * *} & .32(.02)^{* * *} \\ .14(.07)^{*} & -.06(.14) \\ -.07(.02)^{* * *} & -.08(.02)^{* * *} \\ & -.10(.08) \\ & .04(.04) \\ & -.02(.04) \\ & .02(.02)\end{array}$

$\begin{array}{ccc}.14(.04)^{* * *} & .15(.04)^{* * *} \\ 0.40(.02)^{* * *} & .34(.03)^{* * *} & .33(.03)^{* * *} \\ -.11(.05)^{*} & .16(.11) \\ .06(.02)^{* *} & .08(.02)^{* * *} \\ & .09(.06) \\ & -.06(.03) \\ & -.02(.03) \\ & -.03(.01)^{*}\end{array}$

Av. positivity*Negative PDT

Av. negativity*Positive PDT

Av. negativity*Negative PDT

$.02(.02)$

$-.03(.01)^{*}$ 
Positive PDT*Negative PDT

Between-Family

Av. Adjustment (Time 1)

Av. positivity

Av. negativity

Av. positivity*Av. negativity

Random effects

Within-Family

Between-Family

\begin{tabular}{|c|c|c|c|c|c|c|}
\hline 2.116 & $2.039(.07)^{* * *}$ & 2.036 & 1.523 & 1.307 & 1.284 & 1.275 \\
\hline$(.07)^{* * *}$ & & $(.07)^{* * *}$ & $(.05)^{* * *}$ & $(.05)^{* * *}$ & $(.05)^{* * *}$ & $(.05)^{* * *}$ \\
\hline 0.257 & $0.233(.06)^{* * *}$ & 0.227 & 0.458 & 0.139 & 0.125 & 0.125 \\
\hline$(.06)^{* * *}$ & & $(.06)^{* * *}$ & $(.05)^{* * *}$ & $(.04)^{* * *}$ & $(.04)^{* * *}$ & $(.04)^{* * *}$ \\
\hline
\end{tabular}

Model fit indices

AIC

RMSEA

$.12(.04)^{* *}$

$$
\begin{array}{ccc}
.53(.02)^{* * *} & .48(.02)^{* * *} & .48(.02)^{* * *} \\
.27(.06)^{* * *} & .18(.11) \\
-.08(.01)^{* * *} & -.08(.01)^{* * *} \\
& & -.01(.03)
\end{array}
$$

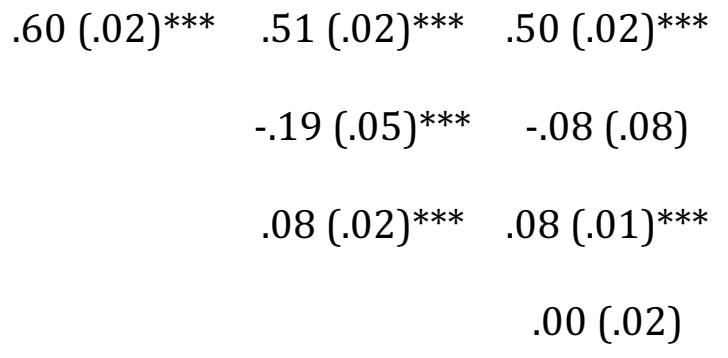


Note: Sex: boy (coded 1), girl (0); Adjustment = within-behavioral domain adjustment at Time 1; CS = child-specific; PDT = parental differential treatment; positivity = mother-child relationship positivity; negativity = mother-child negativity; Av.= family average; ${ }^{*}$ < $.05 ;{ }^{* *} \mathrm{p}<.01 ;{ }^{* * *} \mathrm{p}<.001$ 
Figure 1: Illustrations of interactions between mother-child differential positivity (positive PDT) and mother-child differential negativity (negative PDT) for child prosocial behavior. Note: Upper (UCI) and Lower (LCI) Confidence Intervals.

\section{a) Simple slopes}

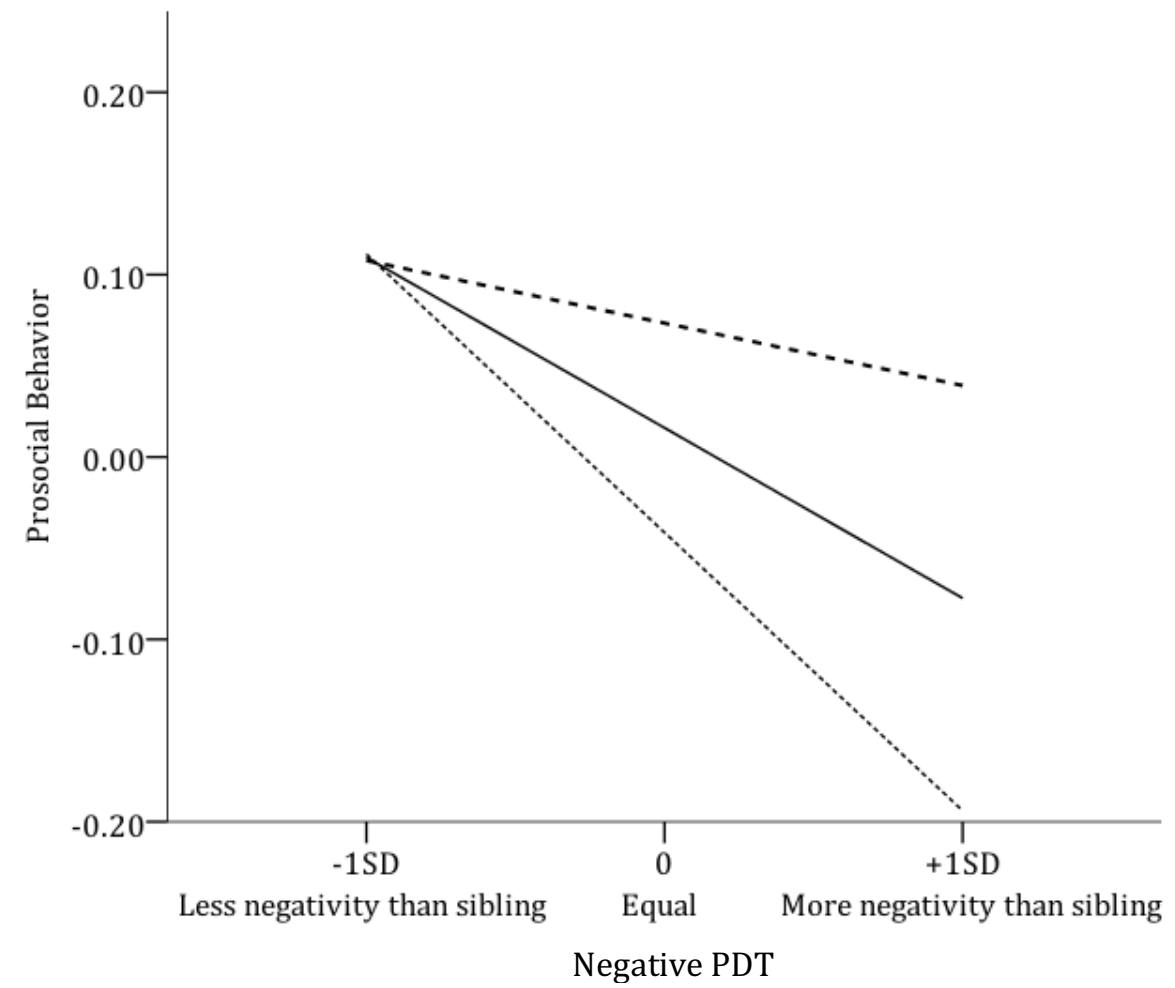

Positive PDT:

..... Less positivity than sibling (-1SD)

- Equal positivity (0)

--- More positivity than sibling (+1SD) b) Johnson-Neyman Regions of Significance

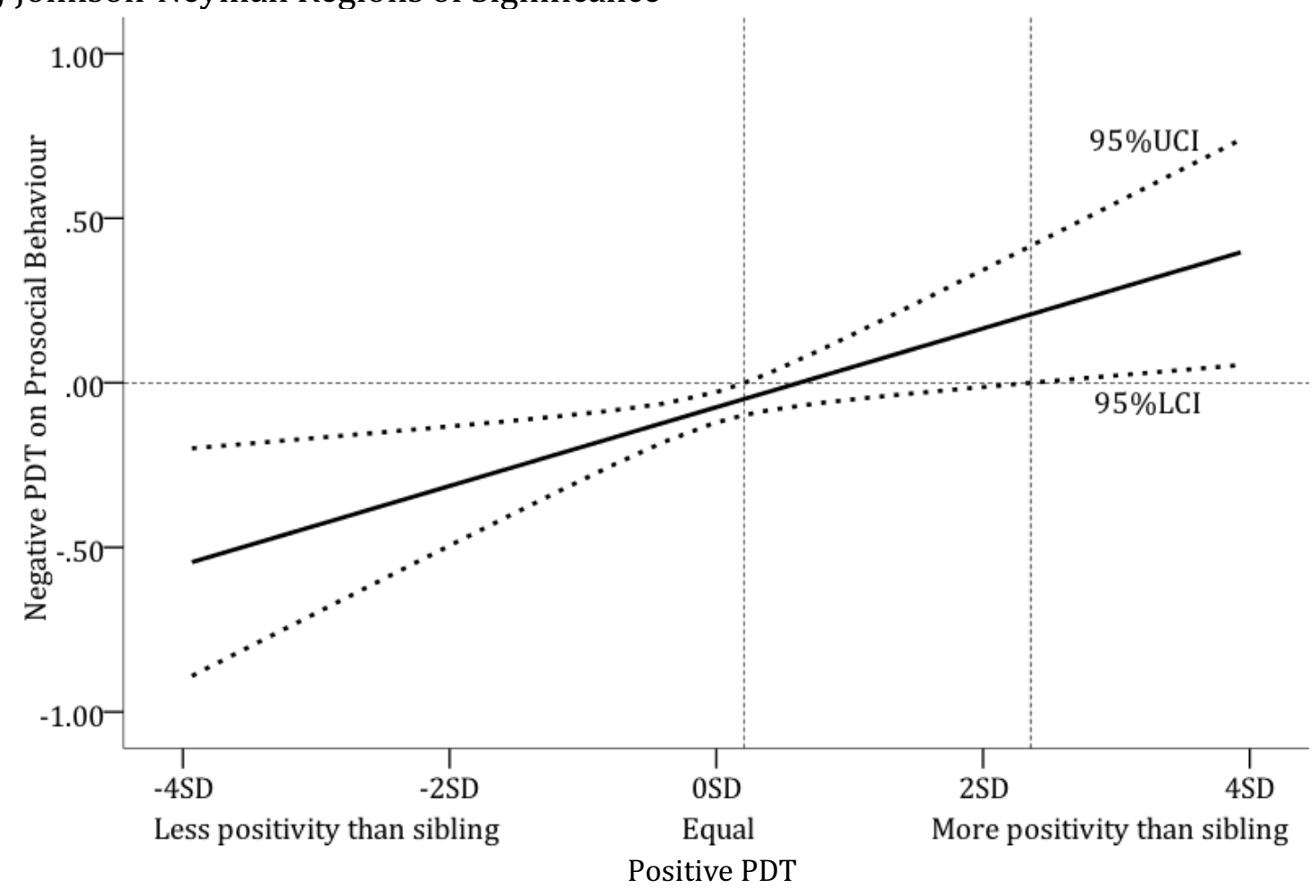


Figure 2: Illustrations of interactions between average (family-wide) mother-child negativity and mother-child differential negativity (negative PDT) for child conduct problems. Note: Upper (UCI) and Lower (LCI) Confidence Intervals.

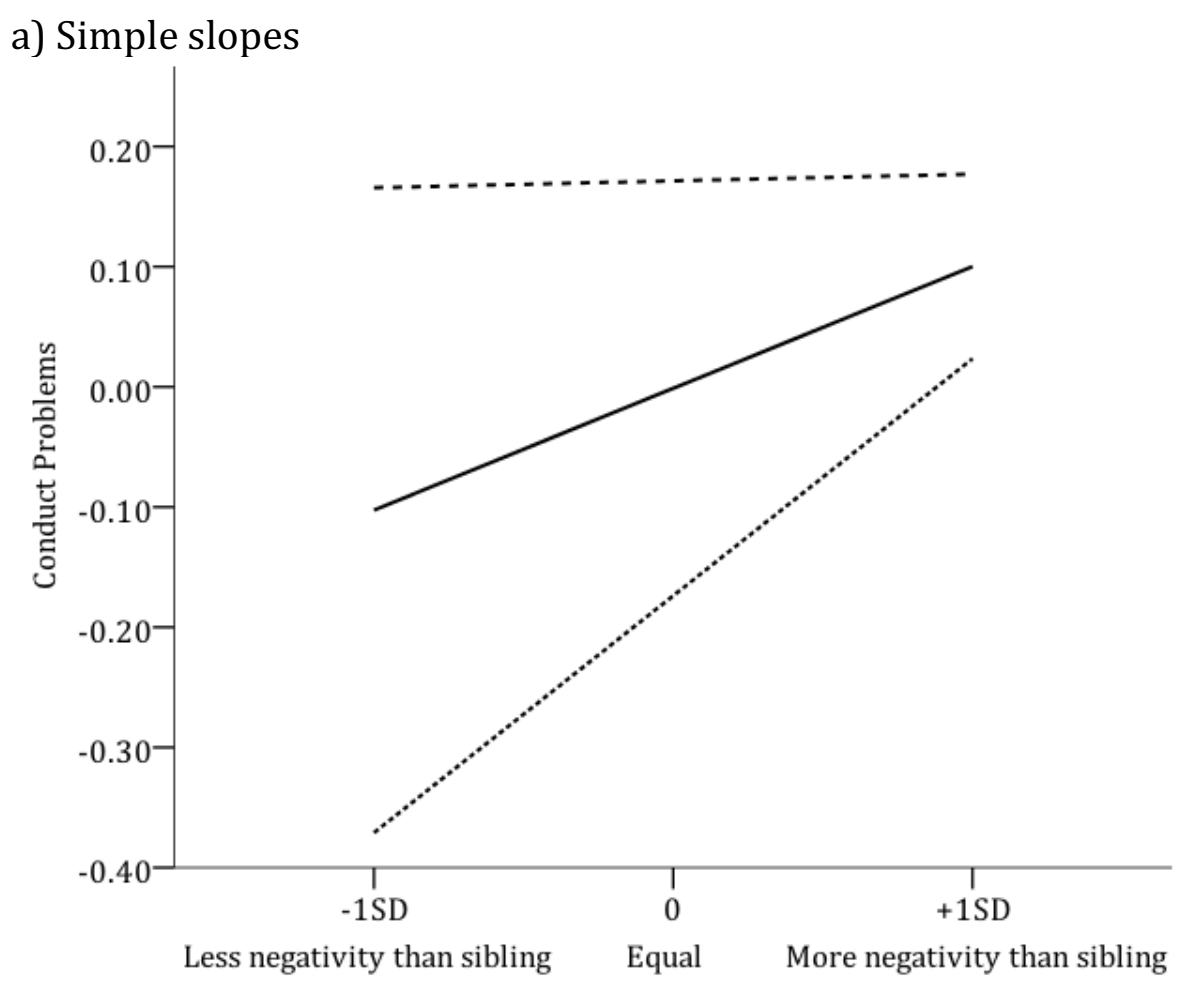

Family-wide Negativity:

.... Low (-1SD)

- Mean (0)

--- High $(+1 S D)$ b) Johnson-Neyman Regions of Significance

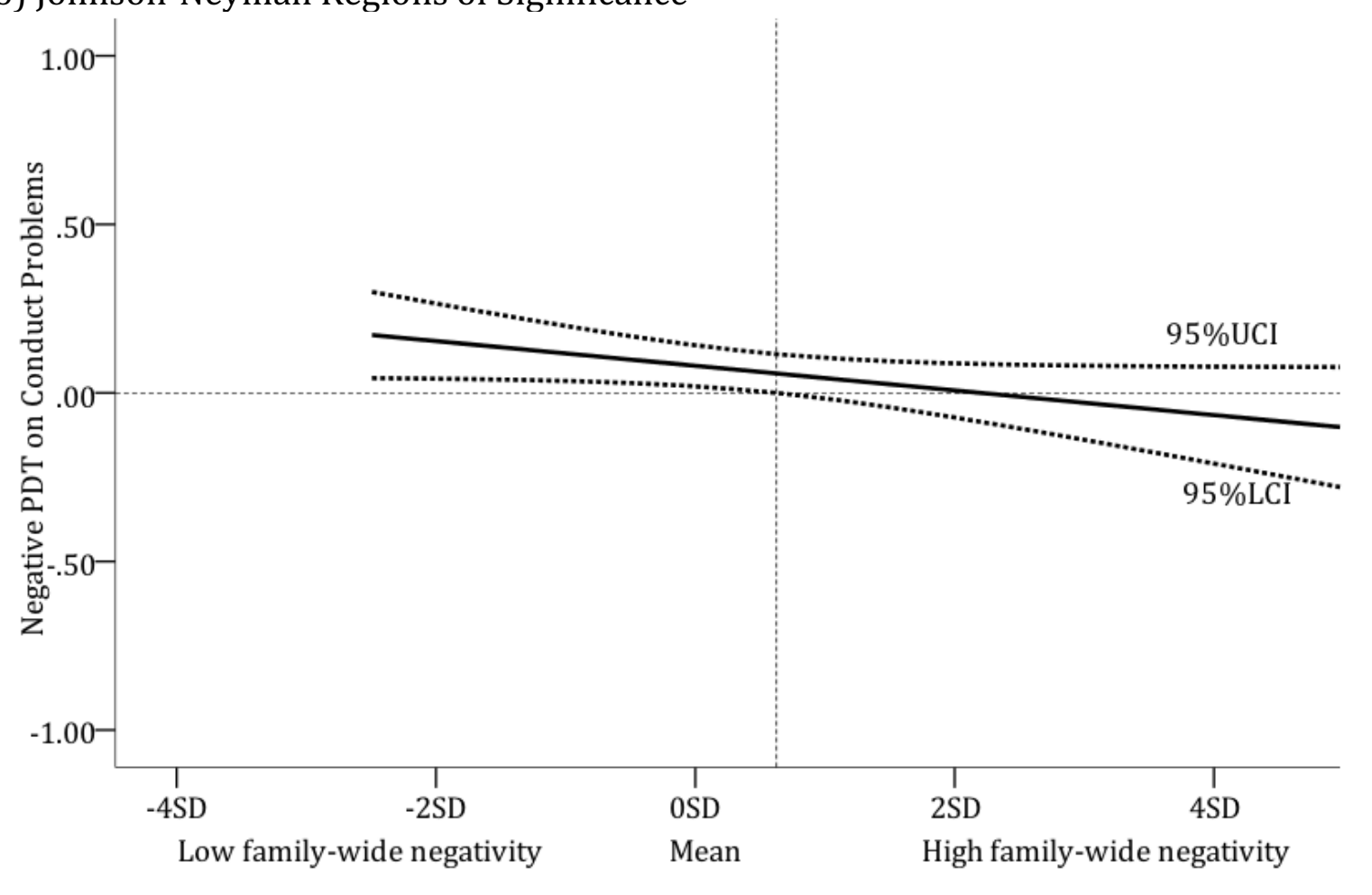

Family-wide Negativity 
Figure 3: Illustrations of interactions between mother-child differential positivity (positive PDT) and mother-child differential negativity (negative PDT) for child conduct problems. Note: Upper (UCI) and Lower (LCI) Confidence Intervals.

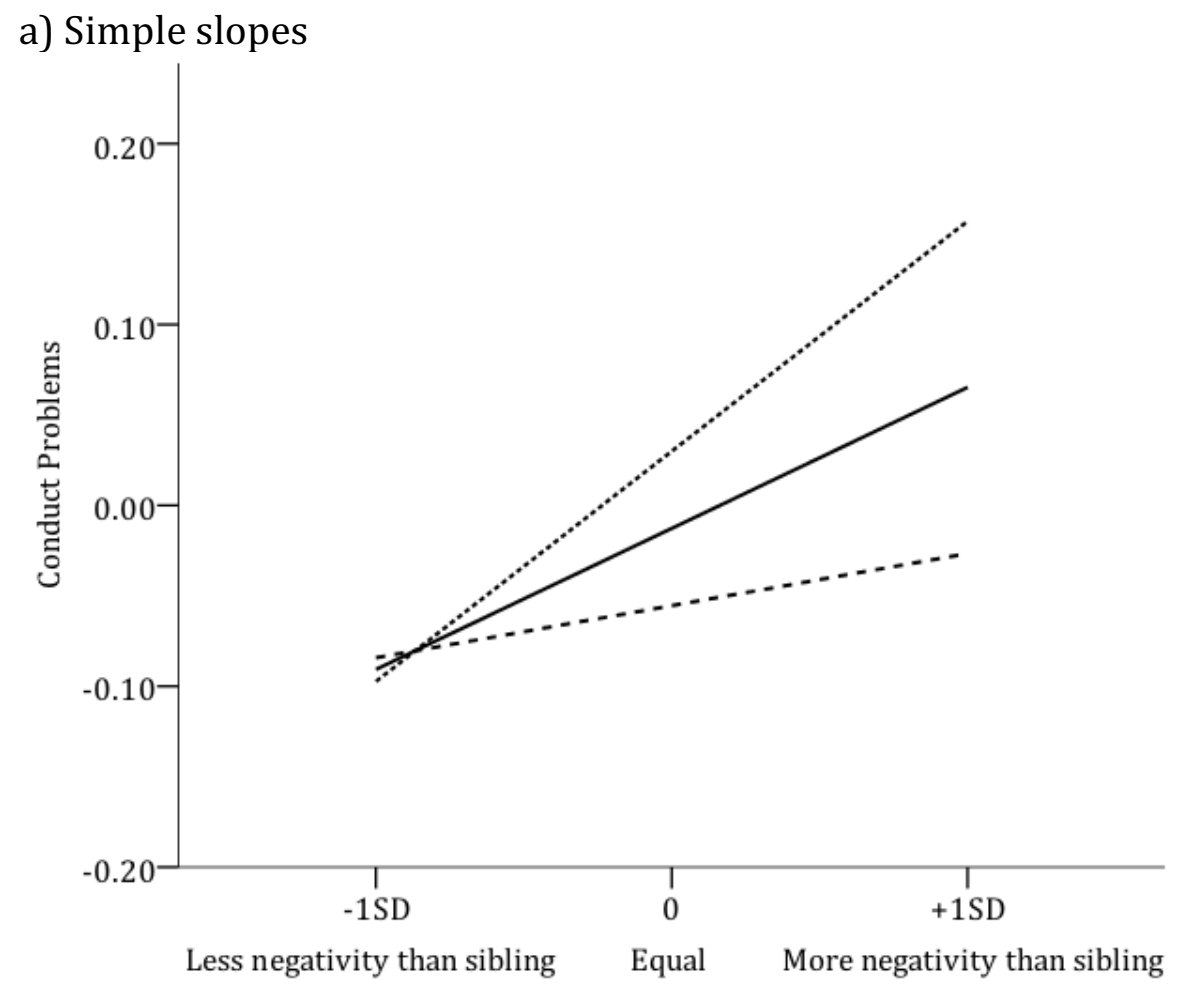

Negative PDT b) Johnson-Neyman Regions of Significance

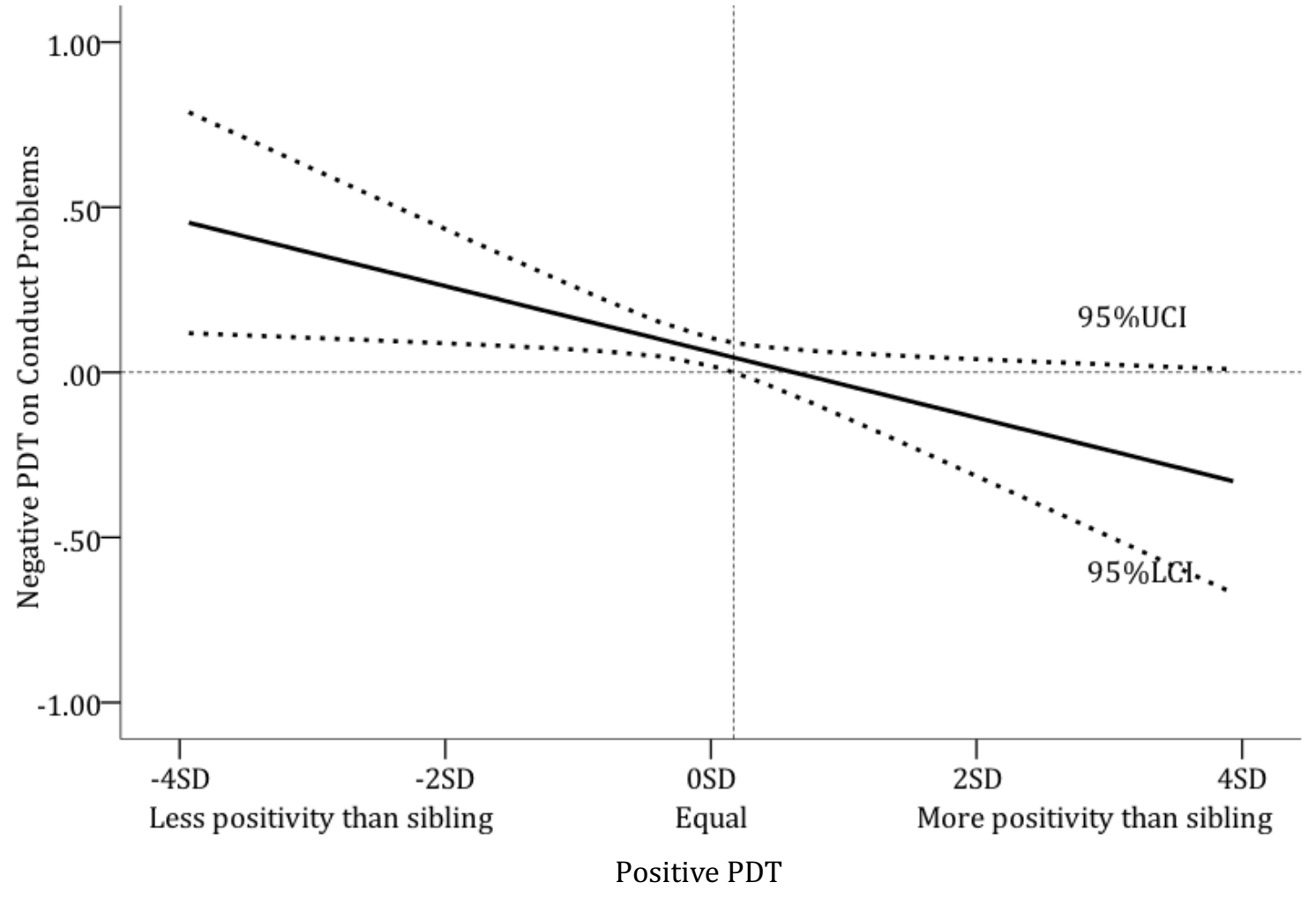

Positive PDT:

.... Less positivity than sibling (-1SD)

- Equal positivity (0)

--- More positivity than sibling (+1SD) 